\title{
The Research on the Distribution Network Reconfiguration and the Application of Immune Particle Swarm Optimization
}

\author{
Zucheng Huang, Lixia Chen, Wei Chen and Xiaojun Chen \\ SouthChinaUniversity of Technology, Haifeng, Guangdong, China, 510000 \\ 2397615891@qq.com
}

\begin{abstract}
With the rapid development of national economy, the demand for electricity is more intense. There is a new demand for the security, reliability and applicability of the power distribution system. The power distribution system is an important component of power systems. It plays an important role in connecting power production and users. Now the most important problem is nonlinear combinatorial optimization in large-scale mixed lines and how to reconfigure the distribution network. However, by deeply analyzing and studying immune particle swarm optimization, the mathematical model of distribution network reconfiguration is established. By coding the characteristics of closed-loop design and open-loop operation, it aims to reduce the consumption of network, balance the grid load, eliminate the current overload and achieve the ultimate goal of improving the power supply reliability.
\end{abstract}

Keywords: distribution network; forward substitution algorithm; network configuration; particle swarm optimization; immune algorithm

\section{Introduction}

The power system consists of four parts: electricity generation, electricity transmission, power distribution and load. Among them, power distribution system is the last link of power system. It is the infrastructure which directly connects with users. It can make the distribution system, power system and power transmission system more closely related with the distribution and supply of power to the user. In order to safeguard the power quality and supply capacity, the analysis and optimization of distribution network which is the most important link in the power distribution system have gradually been a research focus[1]. Distribution network configuration is a complicated large-scale combinatorial optimization problem. Whereas the distribution network configuration studies study from the perspective of the topology, mathematical model, flow calculation method and immune particle swarm optimization method. Through analyzing the topology of distribution network, the paper simplifies the distribution network model distribution network model by using the characteristics of flow calculation method. Through deeply studying particle swarm calculation method and the principle of immune calculation method, the paper finally form a new hybrid algorithm by effectively combining two calculation methods [2]. This is immune particle swarm calculation method. This method mixes respective characteristics of particle swarm calculation method and immune calculation method and can searches and guarantees individual diversity in group, which can improve search ability and accelerate the convergence speed. Minimize the network loss by building distribution network model. And conduct simulation of dynamic configuration and static configuration to distribution network. The result shows that the inherited particle swarm calculation method is effective in the application of distribution network configuration[3]. 


\section{The Research Status Analysis of Distribution Network Reconfiguration}

\subsection{The Research Status of Distribution Network}

Now there have been much research results at home and abroad in the distribution network reconfiguration. Whereas the distribution network research in the early stage mainly studies the planning stage of distribution network. Then, as researchers have a more and more deep understanding, they find that adding network reconfiguration in automated distribution system can not only improve the economy and safety, but also optimize the operation of distribution system at maximum[4].

At present, the optimization methods can be roughly divided into: mathematical optimization algorithm, heuristic algorithm, modern intelligent optimization algorithm etc.

\subsubsection{Mathematical Optimization Algorithm}

Mathematical optimization algorithm describes distribution network reconfiguration through mathematical model. It solves problems by certain mathematical method and gets the optimization results. The distribution network reconfiguration is large-scale network combinatorial optimization. Its biggest problem is that the biggest problem is that the calculation costs a long time and gets too much data. This must will cost huge computer memory and make the distribution network in practical applications face much more difficulties[5]. Generally, when seeking the overall optimal solution by using the method of mathematical optimization, it is necessary to simplify and handle the mathematical model.

\subsubsection{Heuristic Algorithm}

Heuristic algorithm is based on intuitive basis. Generally, it is based on a certain principle. It gradually iterates until the optimization of configuration result reaches best. There are mainly optimal flow pattern algorithm and switches exchange algorithm in distribution network configuration[6].

The final aim of optimal flow pattern algorithm is to guarantee the power consumed least. First, close all the contact switches to form multi-ring network. On the condition of meeting basic loads, disconnect the smallest branch circuit after obtaining the distribution of each branch circuit. This can untie a circuit and recalculate the optimal power flow. It will repeat until the radiation network is formed. Although this simplifies the reconfiguration problem, if there all exist connections between switches, they will influence each other in the initial state of multi-ring network. Thus, the optimal flow method cannot fully guarantee it can get overall optimization in the reconfiguration [7].

The switches exchange calculation method uses heuristic rule, which can not only reduce the switch combination need to be considered, but also determine the reconfiguration result of decrease the distribution wire loss at the fattest speed. However, the method is limited by the initial state of network. Because we can only consider a pair of switch operation, the parallel effect of algorithm is bad.

\subsubsection{Modern Intelligent Optimization Algorithm}

With the international optimal calculation method gradually develops and applies in the optimization field, the application of international optimal calculation method in the reconfiguration of power distribution network is more and more frequently. There are several main algorithms: neutral network algorithm, genetic algorithm, simulated annealing algorithm, ant colony algorithm, particle swarm optimization algorithm etc. Compared with other algorithms, the particle swarm optimization algorithm has no 
processes of choices, crosses, variation etc. So it has the advantages such as high operation speed and good convergence. But because single algorithm has some problems in obtaining overall solution and the calculation of time, the research of integrated optimization algorithm on two or more algorithms attracts more and more attention. For example, combine particle swarm optimal algorithm and other intellectual algorithm and form immune particle swarm algorithm. Simulate plant growth particle swarm algorithm. All these have a lot of achievements[8].

\subsection{The Development Direction of the Reconfiguration of Power Network Distribution}

The reconfiguration of power network distribution is a typical problem in power system. It is often constrained by huge and partial extreme point. It also constrained by the difficulties like discontinuity, multi-dimension, being constrained by flow and nonlinear problems. So, in order to solve these problems and optimal overall solution, we should try to avoid excessive dimensions in case of disasters. Another problem is that power distribution network is the last link of power systems. It is directly connected with users. Its reconfiguration also must guarantee normal load data of users. So the development dimension of the configuration of power distribution network will go to how to get optimal reconfiguration under different loads. In addition, how to improve problem-solving speed reconfiguration. Except some innovations and improvements, it also needs some considerations on the cost of flow calculation. Therefore, another development direction of the reconfiguration of power distribution will be how to find a high-speed effective calculation method which is appropriate to power distribution network on the basis of flow calculation of power distribution network[9].

\section{Immune Particle Swarm Algorithm}

\subsection{The basic Principle of Immune Particle Swarm Algorithm}

\section{(1) The basic Principle of Particle Swarm Algorithm}

Particle swarm algorithm uses a kind of optimal algorithm based on iteration model in continuous space optimization; its number order is described in continuous coordinates:

Setting the particle swarm scale as $\mathrm{N}$, the coordinate position of each particle in $\mathrm{D}$ dimension space can be showed as $\mathrm{X}_{\mathrm{i}}=\left(\mathrm{X}_{\mathrm{I}}, \mathrm{X}_{\mathrm{I}}, \ldots, \mathrm{X}_{\mathrm{Id}}, \ldots, \mathrm{X}_{\mathrm{iD}}\right)$, among which we can define the speed of particle $\mathrm{i}(\mathrm{i}=1,2, \ldots, \mathrm{N})$ as the moving distance of iteration particle. $\mathrm{UseV}_{\mathrm{I}}=\left(\mathrm{V}_{\mathrm{i} 1}, \mathrm{~V}_{\mathrm{i} 2}, \ldots, \mathrm{V}_{\mathrm{id}}, \ldots, \mathrm{V}_{\mathrm{iD}}\right)$ to represent the speed. Thus, we get the flight speed $\mathrm{V}_{\mathrm{id}}$ of particle $\mathrm{i}$ in d dimension space:

$$
\mathrm{V}_{\mathrm{id}} \omega \mathrm{V}_{\mathrm{id}}+\mathrm{c}_{1} \operatorname{rand}_{1}()\left(\mathrm{p}_{\mathrm{id}}-\mathrm{X}_{\mathrm{id}}\right)+\mathrm{c}_{2} \operatorname{rand}_{2}()\left(\mathrm{p}_{\mathrm{gd}}-\mathrm{X}_{\mathrm{id}}\right)
$$

$\omega$ represents inertial factor, $c_{1}, c_{2}$ represent acceleration constant; $p_{g d}$ represents the optimal location of the historical record in the whole particle swarm. Rand ${ }_{1}$ is random generator. It makes certain randomization to the difference between the location of current particle and $p_{g d} \cdot p_{g d}$ represents the optimal location of the historical record for current particle. Similarly available, it also needs random generator makes a certain optimization to the difference between the location of current particle and $\mathrm{p}_{\mathrm{gd}}$.

$$
\text { If } \mathrm{V}_{\text {id }}>\mathrm{V}_{\max } \text {, then } \mathrm{V}_{\mathrm{id}}=\mathrm{V}_{\max } \text {, }
$$

$$
\text { If } \mathrm{V}_{\mathrm{id}}<-\mathrm{V}_{\max } \text {, then } \mathrm{V}_{\mathrm{id}}=-\mathrm{V}_{\max } \text {, }
$$

Through its speed and group cognitive speed, the above formula limits the maximum speed of particle and determines the search accuracy of particle in the solution space. Therefore, when the speed of particle exceeds, then the particle will fly over the optimal solution; instead, if the particle speed is too small, then it can only search in local space rather than comprehensive search in the whole space ${ }^{[10]}$.

The particle can adjust its position through the formula xid=xid+vid. According to the formula, the speed of particle can be understood as the step length of particle towards group 
optimization and itself optimization in d-dimension space.

\section{(2) The basic Principle of Immune Algorithm}

Immune algorithm simulates that when biological antigen intrudes the human body's immune system, the immune system generates corresponding antibody to eliminate the antigen. This algorithm refers to the memory function in immune system, avoids concentration regulation system caused by high concentration of antigen to achieve the possibility of maintaining the diversity of particles and increase the overall convergence.

The particle concentration can be defined with the following formula:

$$
\mathrm{D}\left(\mathrm{x}_{\mathrm{i}}\right)=\mathrm{fi}=1,2, \ldots,(\mathrm{N}+\mathrm{M})
$$

Among them, $\mathrm{f}$ is fitness function, $\mathrm{D}$ ( $\mathrm{xi}$ ) is antibody concentration.

The above formula can deduce the following formula for particle concentration:

$$
\operatorname{PS}(X i)=r_{1} C\left(1-\frac{f\left(x_{i}\right)}{f\left(g_{\text {best }}\right)}\right)+r_{2} \frac{f\left(x_{i}\right)}{f\left(g_{\text {best }}\right)}
$$

Among them, r1, r2 are random numbers between $(0,1)$; $\mathrm{f}(\mathrm{xi})$ is fitness number of particles; $\mathrm{f}$ (gbest) is the largest fitness of particles.

We can draw from the above formula that the ratio between the particle concentration and the probability of selection is inversely proportional. It means that with the increase of particle concentration, the probability of particle to be chosen is less. Instead, low particle concentration is easier to be selected.

\section{(3)Immune Particle Swarm Algorithm}

Although the particle swarm algorithm has the characteristics of quick search speed, simple and easy to implement, it cannot obtain optimal solution because particle swarm algorithm exists convergence in the late stage and falls into local extreme value. Although diversity characteristics of immune algorithm can overcome the defects particle swarm converges. This can effectively adjust the balance between group concentration and individual diversity of particle. It also overcomes the defect of particle swarm algorithm which is easy to fall into local extreme value in late stage.

The flow chart ofimmune particle swarm algorithm is as follows: 


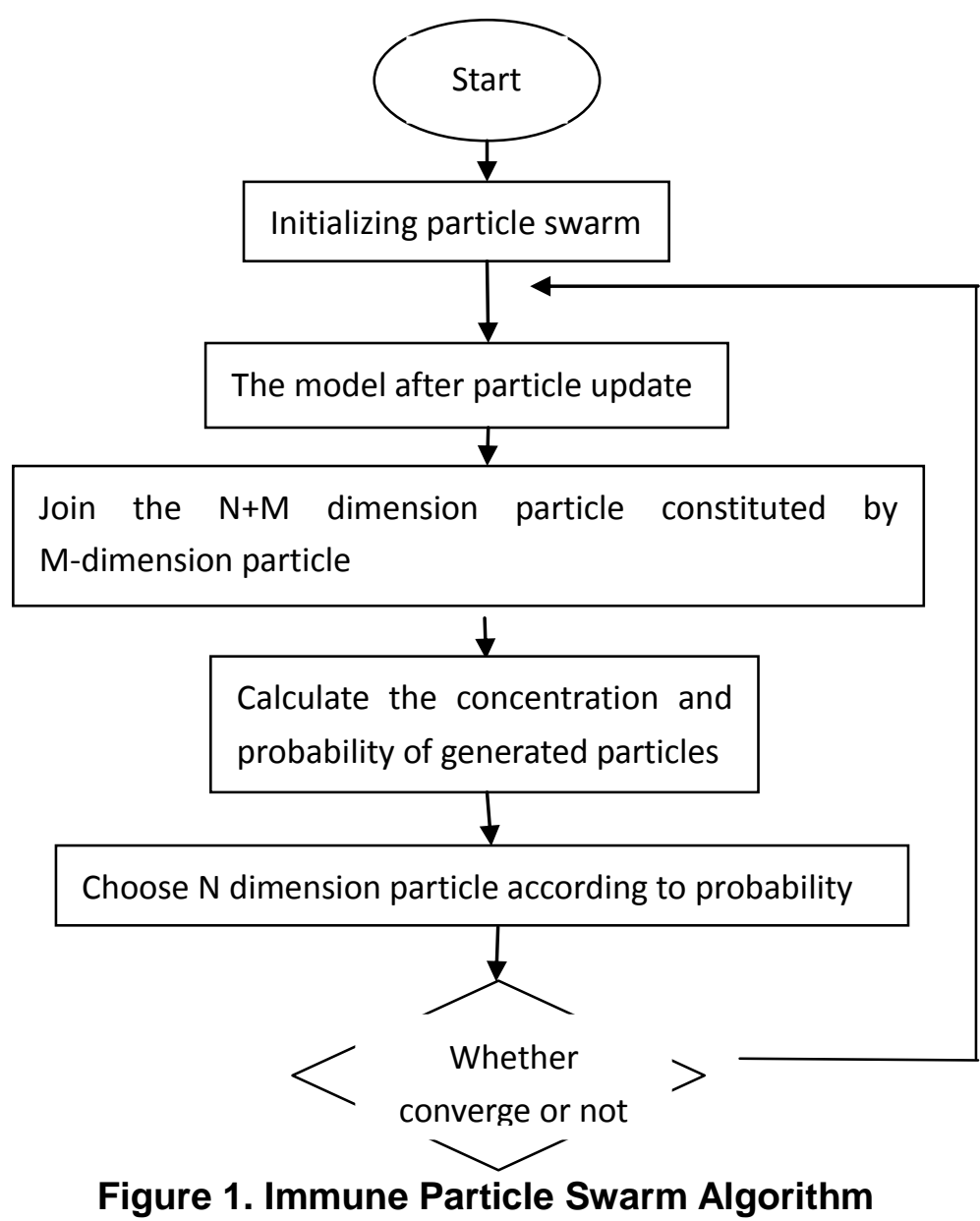

\subsection{Data Storage Mode of Power Distribution Network}

\section{(1) Data Storage Mode of Power Distribution Network}

The calculation of the reconfiguration of power distribution and the data of programming process can be used after special handle. So it needs special handle in the process of data storage in the power distribution network. In practical flow algorithm, we can determine the interrelation between nodes according to the switch status and the first and end points. We can use the tree diagram--node relation matrix to show. For example, the following figure uses 7 nodes to show that:

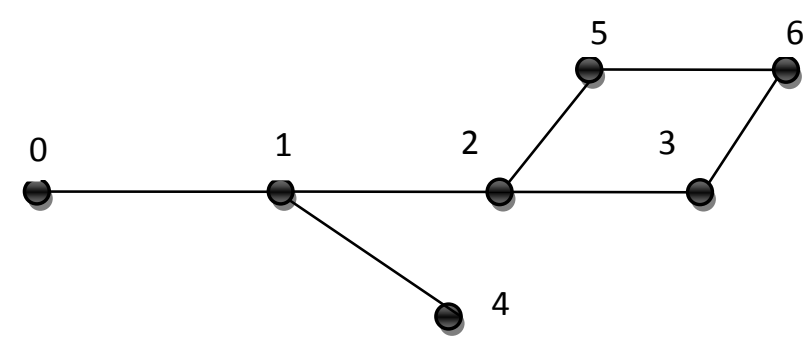

Figure 2.7 Nodes Power Distribution Network

According the above figure, we can determine the topological relations of the network from the original branch structure matrix of 7 nodes network ${ }^{[11]}$. Because the power distribution network is radiation network, so any node only has upstream node to provide 
power energy. According to this energy supply characteristic, we can number the end point of branch and get the results as follows:

$$
\operatorname{Brch}[6][]=\begin{aligned}
& 1 \\
& 2 \\
& 3 \\
& 4 \\
& 5 \\
& 6
\end{aligned}\left[\begin{array}{lll}
0 & 1 & \ldots \\
1 & 2 & \ldots \\
2 & 3 & \ldots \\
1 & 4 & \ldots \\
2 & 5 & \ldots \\
3 & 6 & \ldots
\end{array}\right]
$$

\section{(2) Particle Swarm Storage Mode}

Similarly available, particle swarm algorithm also needs storage in practical application.

Each particle in particle swarm is composed of $\mathrm{N}$ dimension variable which is also called the number of branches. The storage state matrix of each particle can be showed:

$$
\text { Particle }(1)=\left[\begin{array}{llll}
1 & 1 & v_{1}(1) & P_{1}(1) \\
2 & 1 & v_{1}(2) & P_{1}(2) \\
3 & 1 & v_{1}(3) & P_{1}(3) \\
4 & 1 & v_{1}(4) & P_{1}(4) \\
5 & 1 & v_{1}(5) & P_{1}(5) \\
6 & 1 & v_{1}(6) & P_{1}(6) \\
7 & 0 & v_{1}(7) & P_{1}(7)
\end{array}\right]
$$

They are represented: \{branch number, per-dimensional position of particles (switch status group), per-dimensional speed of particles, best position per-dimensional particles $\}$. " 1 "" 0 "the second line if the matrix respectively represent switch on and switch off.

The particle swarm storage matrix in the end is :\{particle number, state matrix of each particle, fitness number (the results of flow calculation), best fitness value of current $\}$, it can be showed:

$$
\operatorname{particleswarm}=\left[\begin{array}{cccc}
1 & \operatorname{particle}(1) & f(1) & f_{P_{\text {best }}}(1) \\
2 & \operatorname{particle}(2) & f(2) & f_{P_{\text {best }}}(2) \\
\ldots & \ldots & \ldots & \ldots \\
\mathrm{M} & \operatorname{particle}(\mathrm{M}) & f(\mathrm{M}) & f_{P_{\text {best }}}(\mathrm{M})
\end{array}\right] \text { (5) }
$$

\section{Simulation Experiment of the Reconfiguration of Power Distribution Network}

This paper chooses IEEE 16 nodes and IEEE 69 nodes in the common test system of power distribution network. It respectively verifies the feasibility and effectiveness of immune particle swarm algorithm in power distribution network proposed in this paper by using the method of static reconfiguration and dynamic reconfiguration. 


\subsection{The Simulation of Static Reconfiguration}

\subsubsection{The Process of Program Design}

The design steps of static reconfiguration algorithm is: first, initiate and input parameters; then, upgrade the network structure according to the upgrading model of particle swarm; next, utilize the network model after upgrade. In order to meet the requirements of flow calculation, we conduct tree method and judge whether it is tree network. If it is a tree network, then we go the next step. On the contrary, we use best value to replace it and go to the next step. Later, we do the flow calculation and calculate active network and assign the numbers. In the end, judge whether it is convergence. If it is convergence, then finish the work and output the results. If not, then continue upgrading the network structure by using the immune particle swarm structure.

\subsubsection{Case Analysis}

This paper simulates and analyzes by using IEEE16 nodes. From the given information, we get that power distribution network has 3 network switches. Among them, its reference voltage is $23 \mathrm{KV}$; its reference capacity is 100MVA. The heavy duty power of whole network node is $28.7+\mathrm{j} 17.3 \mathrm{MVA}$. The simplified diagram of network structure of IEEE16 nodes is as follows:

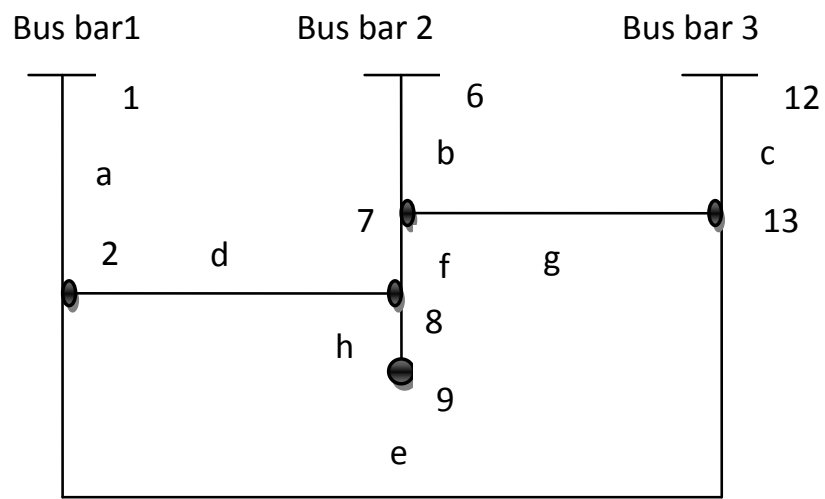

Figure 3. The Simplified Diagram of Network Structure of IEEE16 Nodes

Do initial parameter setting to the system: the number of scale of particle swarm $M=30$, inertia factor $\omega=0.8$, learning factor $\mathrm{c} 1=\mathrm{c} 2=2.0$, the maximum number of iteration is 20 . The maximum number of optimal valueNmax is 5 . The number of generated particles each iteration $F$ is 10 . Switch off the branches 19, 17, 26, the loss of network is $470.327 \mathrm{KW}$. The results before and after reconfiguration are as following chart:

Table 1. Comparison before and after the Power Distribution Network Configuration Network of IEEE16 Nodes

\begin{tabular}{ccc}
\hline \multirow{2}{*}{ Parameters } & \multicolumn{2}{c}{ The state of switches } \\
\cline { 2 - 3 } & Before reconfiguration & After reconfiguration \\
\hline Collection of combined & $5-11$ & $9-11$ \\
switches & $10-14$ & $8-10$ \\
& $1-16$ & $7-16$ \\
Network loss/KW & 511.425 & 470.327 \\
\hline
\end{tabular}


Through compare and analysis, we get that after the optimization of power distribution network, network power loss decreases $8.03 \%$ on the basis of the original. The lowest network voltage of network voltage increases from $0.2 \%$ from $0.9678 \mathrm{p}$.u to present 0.9703 p.u. It can be seen that the reconfiguration of the power distribution network not only has obvious effect, but also improves the lowest voltage of network and the quality of power supply.

\subsection{Simulation of Dynamic Reconfiguration}

\subsubsection{The Design Process of Program Design:}

The design step of the simulation of dynamic reconfiguration is: first, initialization. Input all optimal variables and set parameters of all parameters. Then, do the static reconfiguration during different periods of time and get optimal network structure of this period. Next, combine the periods of time with the same network structure in adjacent periods. Judge whether it meets the requirements of constraint of the number of switches. If it meets the condition, then output the result. If not, continue judging the operation numbers.

\subsubsection{Case Analysis}

The reconfiguration of dynamic network in this paper uses the node algorithm of power network distribution in a certain area. Its reference voltage is $10 \mathrm{KV}$. Reference power is 100MVA. There are 9 nodes and 3 network switches. The structure of power distribution network and network daily load graph are as follows:

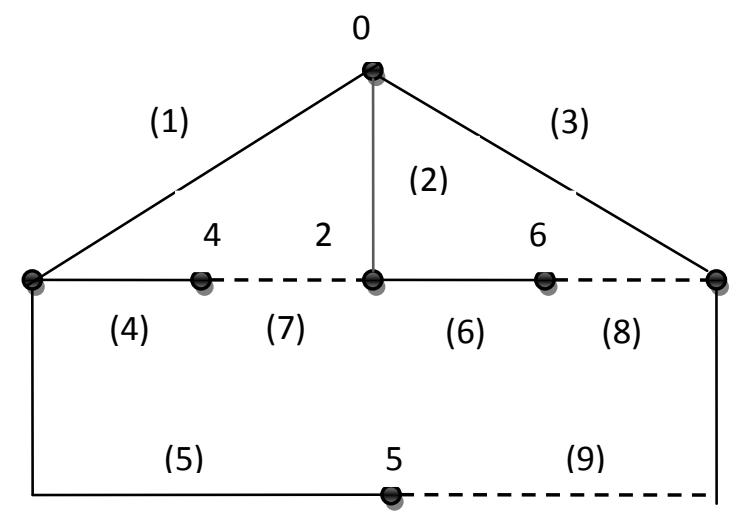

Figure 4. The Structure of Power Distribution Network 


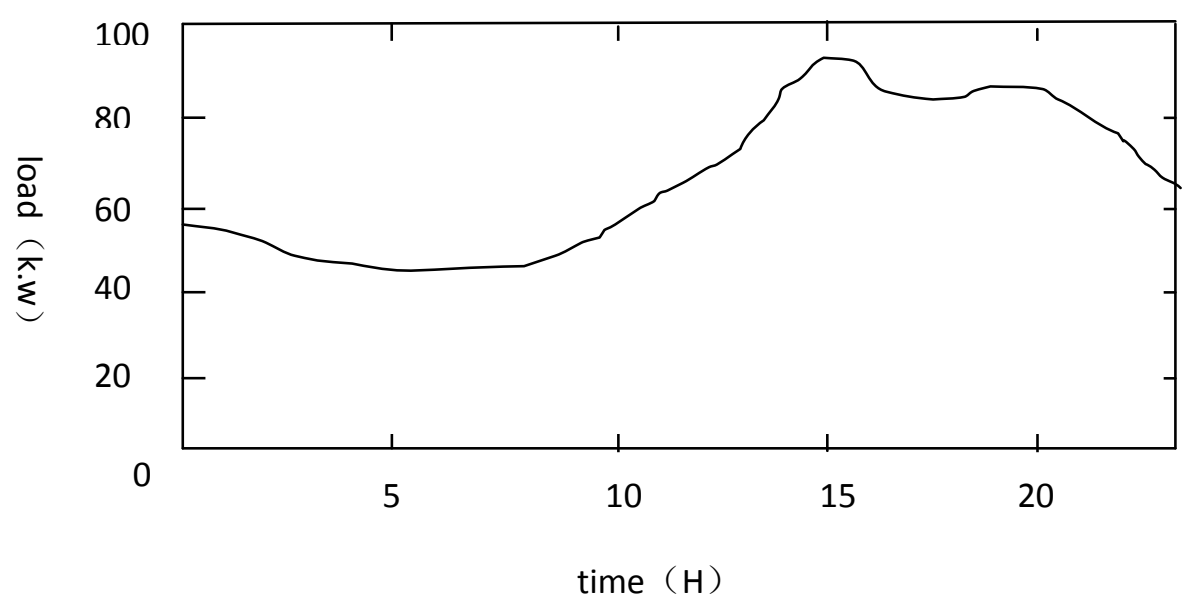

Figure 5. Network Daily Load Graph

In this case, the initial population of particle swarm (M) is 30; the power loss of network is $511.436 \mathrm{~kW}$. The total load is $28700 \mathrm{KW}+\mathrm{j} 17300 \mathrm{KVAR}$. The inertial factor $\omega$ in immune particle swarm algorithm is 0.8. Learning factor $\mathrm{c} 1$, c2 is 2.0. The biggest iteration number is 20 . The maximum number of optimal value Max is 5 . The number of generated particles each iteration is 5 .

First, set the interval of network time as an hour. Conduct a reconfiguration. At the same time, do static reconfiguration to the power distribution network in every period of time by using immune particle algorithm according to known data. the reconfiguration result in every period of time is showed in the following chart:

Table 2. Switch Collection in Every Period of Time after Static Reconfiguration

\begin{tabular}{|c|c|c|c|}
\hline Period of time & $\begin{array}{l}\text { Disconnect the switch } \\
\text { number }\end{array}$ & $\begin{array}{c}\text { the loss before } \\
\text { reconfiguration/KW }\end{array}$ & $\begin{array}{l}\text { The loss after } \\
\text { reconfiguration/KW }\end{array}$ \\
\hline 0 & $5,6,7$ & 1.58 & 1.47 \\
\hline 1 & $5,6,7$ & 1.20 & 1.11 \\
\hline 2 & $5,6,7$ & 1.36 & 1.28 \\
\hline 3 & $5,6 、 7$ & 1.45 & 1.38 \\
\hline 4 & $6 、 7,9$ & 1.25 & 1.12 \\
\hline 5 & $6,7,9$ & 1.10 & 1.01 \\
\hline 6 & $6 、 7,9$ & 1.12 & 1.03 \\
\hline 7 & $6 、 7 、 9$ & 1.09 & 1.03 \\
\hline 8 & $6,7,9$ & 1.89 & 1.78 \\
\hline 9 & $6 、 7,9$ & 3.55 & 3.46 \\
\hline 10 & $6,7,9$ & 4.25 & 4.10 \\
\hline 11 & $6,7,9$ & 4.36 & 4.28 \\
\hline 12 & $6 、 7,9$ & 4.67 & 4.59 \\
\hline 13 & $6 、 7 、 9$ & 4.34 & 4.29 \\
\hline 14 & $6 、 7,9$ & 4.28 & 4.20 \\
\hline 15 & $6 、 7,9$ & 4.20 & 4.14 \\
\hline 16 & $5,6,7$ & 4.15 & 4.04 \\
\hline 17 & $5,6,7$ & 4.02 & 3.87 \\
\hline 18 & $5,6,7$ & 3.80 & 3.71 \\
\hline
\end{tabular}




\begin{tabular}{llll}
19 & $5 、 6 、 7$ & 3.13 & 3.02 \\
20 & $5 、 6 、 7$ & 2.94 & 2.86 \\
21 & $5 、 6 、 7$ & 2.42 & 2.31 \\
22 & $5 、 6 、 7$ & 2.30 & 2.11 \\
23 & $5 、 6 、 7$ & 2.04 & 1.97 \\
\hline
\end{tabular}

The chart lists out the switch number after static reconfiguration in every period of time and the situation of reactive power loss before and after the reconfiguration. We can see from the chart that the power distribution network in every period of time after power distribution network by using immune particle swarm algorithm will decrease.

Next, combine the same network structure in adjacent time from above chart according to a certain rule. The combination result is as follows:

Table 3. Preliminary Segmentation Results

\begin{tabular}{cccccc}
\hline $\begin{array}{c}\text { Para } \\
\text { mete } \\
\text { rs }\end{array}$ & $\begin{array}{c}\text { Dividing } \\
\text { the } \\
\text { period of } \\
\text { time }\end{array}$ & $\begin{array}{c}\text { disconnecting } \\
\text { switch } \\
\text { number }\end{array}$ & $\begin{array}{c}\text { the evaluation of } \\
\text { loss after } \\
\text { reconfiguration/K } \\
\mathrm{W}\end{array}$ & $\begin{array}{c}\text { the evaluation of } \\
\text { loss before } \\
\text { reconfiguration } \\
\text { /KW }\end{array}$ & $\begin{array}{c}\text { Energy } \\
\text { consumptio } \\
\text { n indicator }\end{array}$ \\
\hline 1 & $4-15$ & $6 、 7 、 9$ & 3.23 & 3.06 & 18.20 \\
2 & $16-3$ & $5,6,7$ & 2.16 & 1.99 & 11.78 \\
\hline
\end{tabular}

Through above combination, the network is divided into 2 periods of time. It meets the requirement that the reconfiguration segmentation should not exceed 4 . Then, output the results. The results after the dynamic reconfiguration are as the following chart:

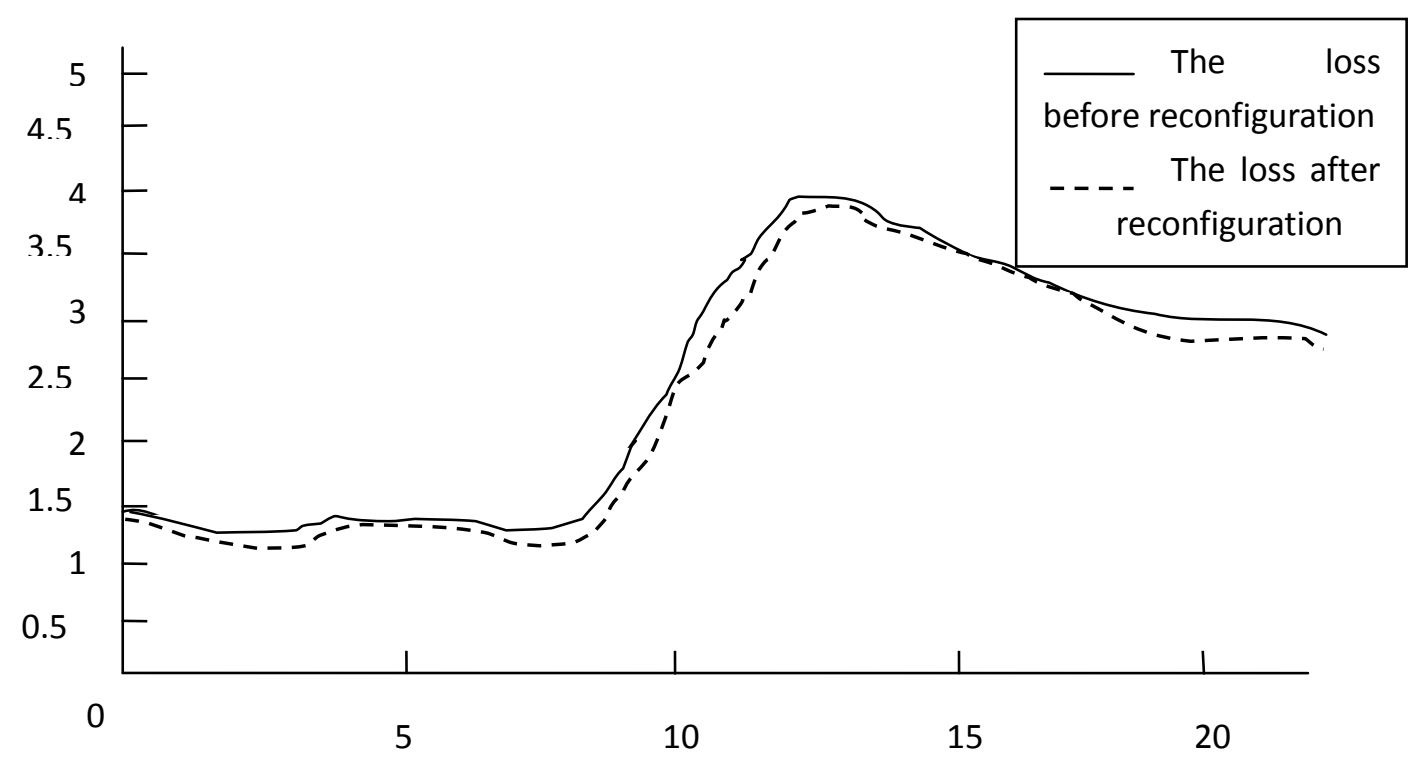

Figure 6. The Power Loss Variation Figure of every Period of Time before and after the Reconfiguration 
Table 4. The Final Results of Dynamic Reconfiguration

\begin{tabular}{cccc}
\hline $\begin{array}{c}\text { The number of } \\
\text { reconfiguration }\end{array}$ & $\begin{array}{c}\text { the state variation of } \\
\text { disconnecting switch }\end{array}$ & Changed switches & $\begin{array}{c}\text { The number of } \\
\text { changed switches }\end{array}$ \\
\hline 1 & $6 、 7 、 9 \rightarrow 5,6,7$ & 5,9 & 2 \\
2 & $5,6 、 7 \rightarrow 6 、 7 、 9$ & 9,5 & 2 \\
\hline
\end{tabular}

According to the data from above, we can get the conclusion that the active power of the reconfiguration of power distribution network decreases $5.7 \%$ from $2.65 \mathrm{KV}$ to $2.50 \mathrm{KV}$. The dynamic reconfiguration of immune particle swarm algorithm used by this paper effectively reduces the loss of active power network and decreases the complexity of the reconfiguration of power distribution network at maximum.

\section{Conclusion}

This paper mainly studies the optimal algorithm which combines the immune algorithm and particle swarm algorithm, proposes the immune particle swarm algorithm. Establishes the simulation model of power distribution network with static reconfiguration and dynamic reconfiguration, simplifies the power distribution network by using the method of graph theory. This narrows the space range of solution and eliminates much invalid solution so as to improve the search efficiency of algorithm. Finally, the purpose is to reduce the number of active power loss by simulation, to reduce the complexity of the reconfiguration and improve reconstruction efficiency. Because of the limitation of time and level, the paper doesn't involve the situation such as the quality of power supply, unknown load. So there still needs a lot of improvements.

\section{References}

[1] Jorge Mendoza, Rodrigo Lopez, Dario Morales, et al. Minimal loss reconfiguration using genetic algorithms with restricted Population and addressed operator's real application. IEEE Transaction on Power System S[J], 2006, 21(2): 948 954

[2] Wen Zhang, Yutian Liu. Fuzzy Logic Controlled Particle Swarm for Reactive Power Optimization Considering Voltage Stability. The 7th International Power Engineering Conference, 2005

[3] Morton.A.B, Mareels.I.Y. An Efficient Brute-Force Solution to the Network Reconfiguration Problem, IEEE Trans on Power Delivery, 2000, 15(3):996-1000

[4] Fan Ji-Yuan, Zhang Lan, Mc Donald J, Distribution Network Reconfiguration: Single Loop Optimization, IEEE Trans on Power Systems, 1996, 11(3):1643 1647

[5] Marcos A. N. GuimarZIes, Jorge E C. Lorenzeti, Carlos A.Castro. Reconfiguration of distribution systems for stability margin enhancement using tabu search, 2004 International Conference on Power System Technology. (Singapore. November 21 23, 2004)

[6] Ching-Tzong Su, Chung-Fu Chang, Ji-PyngChiou. Distribution Network Reconfiguration for loss reduction by Ant Colony Search Algorithm [J].On Electric Power Systems Research. 2005, 190-199

[7] F van den Bergh. An Analysis of Particle Swarm Optimizers [D], Ph D thesis, Department of Computer Science, University of Pretoria, South Africa, 2002

[8] M.A.Kashem, VGanapathy, G.B.Jasmon. Network reconfiguration for enhancement of voltage stability in distribution networks, IEE Proc. - Gener, Transm. Distrib, Vol.147, No.3, May 2000

[9] F.V.Gomes, S.Carneiro, J.L.R.Pereira.A new distribution system reconfiguration approach using optimal power flow technique and sensitivity analysis for loss reduction. IEEE PES General Meeting, 2005, Vol.1:897-901

[10] Ching-Tzong Su, Chung-Fu Chang, Ji-PyngChiou. Distribution Network Reconfiguration for loss reduction by Ant Colony Search Algorithm [J]. On Electric Power System Research.2005, 190 199

[11] Young-Jae Jeon, Jae-chul Kim. An eifficient simulated annealing algorithm for network reconfiguration in large-scale distribution systems. IEEE Trans on Power Delivery, 2002, 


\section{Author}
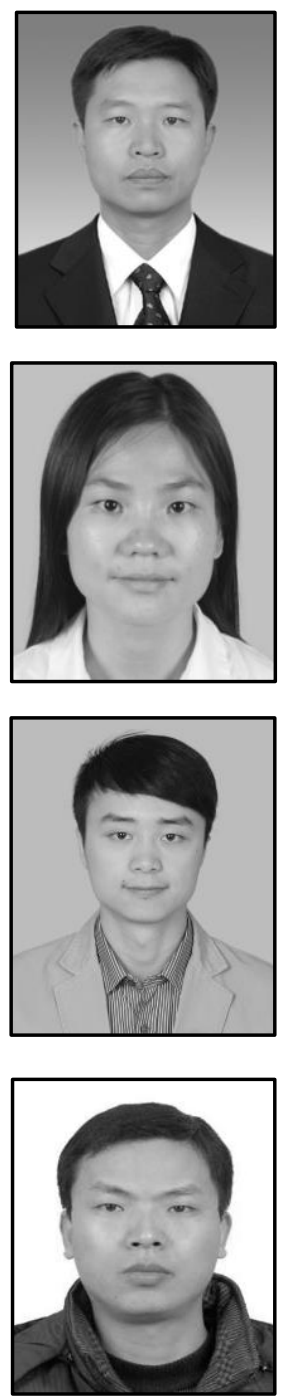

Zucheng Huang, He is a master of South China University of Technology and works in the Guangzhou Power Supply Bureau Ltd as a senior engineer. The address is No. 2656 Huangfu Power Supply Bureau, Kaichuang Road, Guangzhou, Guangdong Province.

Lixia Chen, She has a bachelor of South China University of Technology and works in the Guangzhou Power Supply Bureau Ltd. The address is No. 2656 Huangfu Power Supply Bureau, Kaichuang Road, Guangzhou, Guangdong Province.

Wei Chen, He is a master of Sanxia University and works in the Guangzhou Power Supply Bureau Ltd. The address is No. 2656 Huangfu Power Supply Bureau, Kaichuang Road, Guangzhou, Guangdong Province.

Xiaojun Chen, is a college student of National Defense University and works in the Zhuhai Hua Wei Electricity Co Ltd. The address is 4th floor unit 5-8 in southern software park B5 building in Xiangzhou District in Zhuhai. 